\title{
A Proposal of Educational Video Gamification as a Service
}

\author{
Daniel de Sousa Moraes \\ danielmoares14@gmail.com \\ UFMA, São Luís, Brazil
}

\author{
Álan L. V. Guedes \\ alan@telemidia.puc-rio.br \\ PUC-Rio, Rio de Janeiro, Brazil
}

\author{
Antonio J. G. Busson \\ busson@telemidia.puc-rio.br \\ PUC-Rio, Rio de Janeiro, Brazil
}

\author{
Carlos de Salles Soares Neto \\ csalles@deinf.ufma.br \\ UFMA, São Luís, Brazil
}

\author{
Sérgio Colcher \\ colcher@inf.puc-rio.br \\ PUC-Rio, Rio de Janeiro, Brazil
}

\begin{abstract}
Online and hybrid courses are characterized by the use of technologies teaching support and student-centered methodologies. In particular, the use of content authoring, storage, distribution, and presentation technologies has contributed to the emergence of Virtual Learning Environments (VLEs), such as Moodle, and MOOCS (Massive Open Online Course), such as EdX. However, those types of courses still suffers from problems involving student engagement. To improve this engagement, this article discusses the proposal of a Deep Learning as a Service (DLaS) called EVGAS (Educational Video Gamification As a Service). More precisely, EVGAS is a service for recommending and gamifying activities in existing educational VLE videos. First, EVGAS uses Deep Learning techniques to classify videos from an AVA. Then, it accesses repositories of activities, such as Khan Academy and UVA OnlineJudge, in order to select activities according to the topics classified from the videos. Finally, EVGAS adds VLA activities and gamification. It allows the teacher to monitor student progress, including in relation to each topic of the video. As a result, this paper presents high level requirements and an EVGAS Mockup.
\end{abstract}

\section{KEYWORDS}

video analyses, educational video, gamification, datasets, neural networks

\section{INTRODUÇÃO}

O paradigma tradicional de cursos presenciais centrados no professor tem dado lugar a cursos on-line ou híbridos. Tais cursos se caracterizam pelo uso de tecnologias para apoio ao ensino e de metodologia centrada no estudante [3]. Nesse contexto, o uso de tecnologias de autoria, armazenamento, distribuição e apresentação de conteúdo contribuíram para o surgimento de Ambientes Virtuais de Aprendizagem (AVAs) como o Moodle e MOOCS (Massive Open Online Course). Entretanto, esse novo paradigma ainda sofre de problemas envolvendo o engajamento dos alunos [11].

Segundo Klock et. al.[6], alguns fatores que contribuem para problemas de engajamento são a falta de compreensão do plano de ensino, falta de confiança, medo ou a didática utilizada. Em particular, alguns estudos [8] indicam que existe uma relação entre o engajamento do aluno com seu rendimento e desenvolvimento

In: VI Workshop "O Futuro da Videocolaboração" (WCT-Video 2019), Rio de Janeiro Brasil. Anais Estendidos do Simpósio Brasileiro de Sistemas Multimídia e Web (WebMedia). Porto Alegre: Sociedade Brasileira de Computação, 2019.

ISSN 2596-1683 cognitivo e social. Uma estratégia que tem sido utilizada para promover engajamento é a gamificação [5]. Ela utiliza características de jogos digitais para o ensino como ranking e badges. Este trabalho busca adicionar elementos de gamificação para atenuar esses problemas de engajamentos em AVAs. Nesse sentido, discutimos a seguir Atividades em ambientes educacionais e Deep Learning para vídeo. Um elemento importante na gamificação é adição de atividades que podem ser utilizados como desafios para os alunos. Utilizar atividades em um AVA permite o uso de pontuações, badges e tabelas de ranking. A Figura 1 ilustra uma atividade com um quiz feito no ambiente Moodle. A criação desse tipo de atividade é dispendiosa e requer um esforço do professor.

Outras plataformas de ensino, não necessariamente AVAs, buscam oferecer suporte à resolução de atividades por alunos. A Figura 2 mostra um quiz da plataforma Khan Academy. Existem casos de professores que adicionam links de atividades nesses outros ambientes em seus AVAs. Entretanto essa ação é feita manualmente e não é integrada aos AVAs.

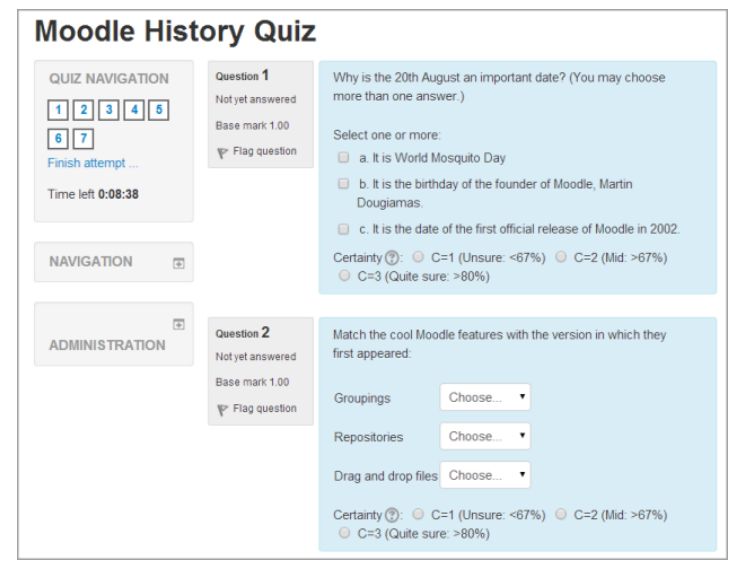

Figura 1: Atividade no Moddle ${ }^{1}$

As atividades desses repositórios poderiam ser utilizadas para facilitar a gamificação de um curso por professores. Neste artigo, temos o objetivo de auxiliar o professor na gamificação através da recomendação de atividades desses repositórios Para realizar essa recomendação, propomos o EVGAS (Educational Video Gamification As a Service), que primeiro realiza uma classificação de vídeos utilizados pelo professor no AVA e em seguida recomenda atividades relacionadas ao conteúdo dos vídeos, encontradas em um banco de repositórios.

\footnotetext{
${ }^{1}$ https://docs.moodle.org/37/en/Assignment_activity
} 


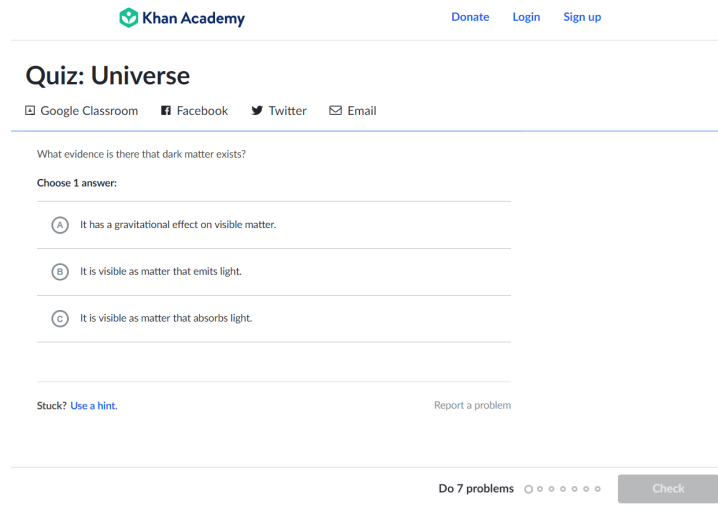

Figura 2: Quiz no Khan Academy

Nos últimos anos, o Deep Learning (DL) permitiu significativo avanço de vários segmentos da multimídia, principalmente em tarefas relacionadas a processamento de fala, audição e visão computacional [10]. Em particular, as arquiteturas de DL baseadas em CNNs (Convolutional Neural Network), ou ConvNets, se tornaram o principal método usado para reconhecimento de padrões audiovisuais. Plataformas como IBM Watson ${ }^{2}$ e Microsoft Azure $\mathrm{ML}^{3}$ já oferecem DLaS (Deep Learning as a Service), permitindo que sistemas multimídia possam incorporar novas funcionalidades baseadas em aprendizagem e reconhecimento de padrões.

Nesse artigo, propomos o EVGAS (Educational Video Gamification As a Service) como um serviço para gamificação atividades em vídeos educacionais presentes em AVAs. Ele utiliza tanto técnicas de gamificação quanto de classificação de vídeo com Deep Learning. O serviço é o responsável pela classificação da videoaula e extrai os possíveis tópicos abordados. Em seguida, ele recomenda atividades para ser utilizadas pelo professor para a gamificação.

Para apresentar esta proposta, o restante deste artigo está organizado como segue. A Seção 2, traz o estado da arte em Gamificação. Enquanto a Seção 3 apresenta o estado da arte em tarefas de classificação de vídeo. A seguir é detalhada a presente proposta de serviço na Seção 4. Por fim, são apresentadas as considerações finais e trabalhos futuros na Seção 5.

\section{ESTADO DA ARTE EM GAMIFICAÇÃO}

Segundo Deterding et al. [1], gamificação (do inglês: Gamification) refere-se ao emprego de técnicas e elementos utilizados no desenvolvimento de jogos, em contextos sem relação com jogos. Já Kapp [5], diz que gamificação é a incorporação de elementos de jogos em sistemas interativos não-jogo e/ou a aplicação de elementos de jogos, como mecânica, dinâmica e estética em contextos não relacionados a jogos. O principal motivo para o uso da gamificação é, segundo os autores citados anteriormente, motivar um melhor engajamento dos usuários para a execução das atividades do sistema ou ambiente em questão.

$\mathrm{Na}$ tentativa de explorar o potencial motivacional da gamificação, diferentes áreas como turismo, gestão, assistência médica e educação têm buscado as melhores formas de aplicação de suas técnicas na solução de problemas de engajamento e desempenho de seus públicos [1].

\footnotetext{
${ }^{2}$ https://www.ibm.com/watson

${ }^{3}$ https://azure.microsoft.com/pt-br/services/machine-learning-studio
}

A gamificação tem sido utilizada essencialmente para alterar a relação homem-máquina, tornando-a mais envolvente e atrativa. Deterding et al. [1] ainda constata que a gamificação usa o design e os elementos característicos para jogos em contextos de não-jogos, o que a diferencia de outros conceitos relacionados que estendem esse design ou usam tecnologias baseadas em jogos ou outras práticas de jogos, e que incorporam jogos completos ao invés de apenas elementos característicos de jogos, independentemente das intenções do contexto.

Em educação, o uso de jogos incentiva o engajamento dos alunos pelo fato de permitir que estes reiniciem, joguem novamente e cometam erros que são recuperáveis. Segundo Lee e Hammer [7], essa liberdade permite que os alunos experimentem sem medo de falhar e consequentemente, aumentando seu empenho. Outra vantagem é o feedback mais frequente e imediato que pode ser emitido pelo game design, sendo muito mais benéfico para os professores que geralmente podem avaliar e dar feedback a apenas um aluno por vez, levando-se em conta as limitações naturais dos professores, tais avaliações tomam bastante tempo [5].

Além disso, uma vez que professores tradicionalmente apresentam as informações aos alunos baseadas em sua dificuldade, a gamificação pode ser usada para personalizar a progressão de cada indivíduo no aprendizado de uma determinada disciplina, mantendo o aluno em um determinado nível até que este demonstre que de fato dominou o assunto e pode seguir adiante.

Em [12], os autores definem sete elementos primários para a gamificação, sendo eles: conquistas, pontuação, níveis, tabelas de ranking, distintivos (badges), desafios/missões, engajamento inicial e demais ciclos de engajamento. Eles defendem que a ideia principal é permitir que o "jogador" utilize estímulos intrínsecos (competição e cooperação) e extrínsecos (níveis, pontuações, missões) para realizar as atividades propostas. Já Kapp [5] define que a gamificação pode se utilizar de todos os elementos de jogos que sejam apropriados para promover o engajamento e a aprendizagem, desde que estes elementos estejam interconectados entre si e com o contexto em que está inserido.

\section{ESTADO DA ARTE DE CLASSIFICAÇÃO VÍDEO}

Em Multimídia, a tarefa de classificação consiste em mapear um conteúdo de mídia em uma ou mais categorias distintas. Arquiteturas de Deep Learning baseadas em CNNs (Convolutional Neural Network) ou ConvNets se tornaram o principal método usado para reconhecimento de padrões audiovisuais. Tipicamente o treinamento de CNNs é feito de maneira supervisionada, e são treinadas em datasets que contém milhares/milhões de mídias e classes relacionadas. Durante o treinamento, as CNNs aprendem a hierarquia de features que são aplicadas a mídia de entrada para que seja possível realizar a classificação do seu conteúdo.

Desde a vitória da CNN AlexNet no desafio ImageNet 2012, surgiram novas arquiteturas baseadas em $\mathrm{CNN}$ cada vez mais precisas. A vencedora do ImageNet 2014, por exemplo, foi a CNN InceptionNet, que propôs o uso do bloco Inception. Esse bloco usa vários filtros de diferentes tamanhos no mesmo nível para resolver o problema de localização da informação em imagens. No ano seguinte, a rede ResNet foi a vencedora do ImageNet 2015, e introduziu o conceito de conexões residuais, que aumentou a performance e reduziu o 

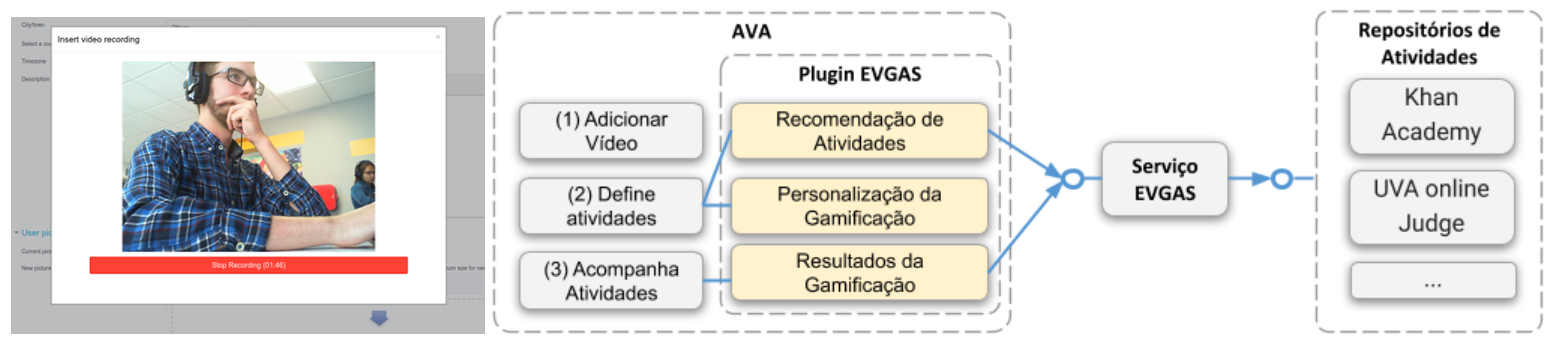

Figura 3: EVGAS integrado ao Moodle

tempo de treinamento das CNNs. Mais tarde foi desenvolvida a arquitetura Inception-Resnet, , que combina os blocos Inception com as conexões residuais. Essa arquitetura é popular e é a base para muitas outras arquiteturas de $\mathrm{CNN}$ para extração de features.

A arquitetura SE-Net (Squeeze-and-Excitation Network) [4] é o estado-da-arte na tarefa de classificação de imagens, obtendo $2.25 \%$ de erro top-5 no ImageNet 2017. Assim como o InceptionNet, a SE-Net propõe um novo tipo de bloco chamado SE, que melhora o poder de representação da rede ao destacar as interdependências entre os canais da imagem e seus mapas de features. Para isso, a SE-Net usa um mecanismo que permite que a rede possa fazer uma recalibração de features, através do qual, usa informações globais para enfatizar as features mais informativas e suprimir as features menos úteis.

Diferente de imagens, vídeos não possuem apenas informação visual, mas também auditiva. Métodos atuais para classificação de vídeo geralmente representam os vídeos pelas features audiovisuais extraídas dos seus quadros, seguido pela agregação dessas features sobre o tempo. Métodos usados para extração de features incluem modelos de CNN pré-treinados em datasets de larga escala. O YouTube $8 \mathrm{M}^{4}$, por exemplo, usa a rede Inception-Resnet pré-treinada no ImageNet para extrair features visuais, e usa a rede Audio-VGG [2] pré-treinado no Audioset para extrair features auditivas. Já métodos para agregação de features incluem métodos sofisticados de pooling como na CNN NetVLADou usando modelos recorrentes baseados em GRUou LSTM. Em particular, a arquitetura Gated-NetVLAD [9] conseguiu obter $84 \%$ de GAP no desafio de classificação chamado Youtube $8 \mathrm{M}$ em 2017. Esse desafio utiliza um dataset que contém mais de 6 milhões de vídeos distribuídos em 3862 classes de etiquetas.

\section{EVGAS}

Técnicas de gamificação podem aumentar o engajamento de alunos. Entretanto, a aplicação dessas técnicas requer um esforço significativo dos professores. Para auxiliar na gamificação, este trabalho propõe a ferramenta EVGAS. Essa ferramenta facilita o uso de técnicas de gamificação em ambientes virtuais de aprendizagem como o Moodle. Mais precisamente, o EVGAS se utiliza de técnicas Machine Learning para recomendar atividades para professores utilizarem na gamificação dos seus cursos.

Para realizar a recomendação, o EVGAS faz a classificação de vídeos utilizados pelos professores. Por exemplo, a Figura 3 (na parte mais à esquerda) ilustra a funcionalidade do Moodle que permite o professor gravar uma aula e carregar diretamente ao ambiente. Esses

\footnotetext{
${ }^{4}$ https://research.google.com/youtube8m/
}

vídeos são classificados pelo EVGAS para permitir a recomendação de atividades e a gamificação. Para detalhar a funcionalidades do EVGAS, discutimos a seguir requisitos de alto nível da ferramenta:

- RQ1. Classificar o vídeo segundo ontologia de tópicos de ensino: permitir a seleção dos tópicos para busca de atividade dentre uma lista de tags resultante da classificação da videoaula.

- RQ2. Recomendar atividades segundo os tópicos de um vídeo: fornecer uma lista de atividades recomendadas dos repositórios segundo os tópicos selecionados.

- RQ3. Personalizar Gamificação: propiciar a escolha dos elementos de gamificação para utilização assim como o ajuste dos parâmetros destes, conforme a necessidade.

- RQ3. Acompanhar os resultados das atividades: permitir a visualizar do progresso dos alunos na resolução das atividades nos repositórios, identificando os resultados alcançados por estes em cada tópico abordado.

Para implementar esses requisitos, propomos uma arquitetura do EVGAS organizada em dois componentes como ilustra a Figura 3. Os componentes são: o (1) Plugin EVGAS; e o (2) Serviço EVGAS. O Plugin deve ser incorporado aos AVAs. Ele visa permitir a adição de atividades correlacionadas aos temas das videoaulas. Além disso, ele também deve permitir que o professor personalize a gamificação e acompanhe os resultados dos alunos. Já o Serviço visa a classificação da videoaula, busca e recomendação das atividades. Além disso, ele deve buscar os resultados dos alunos na resolução das atividades. Detalhamos esses dois componentes nas subseções a seguir.

\subsection{Plugin EVGAS}

O plugin auxilia o professor no processo de gamificação da aula. Esse processo é ilustrado na Figura 3. No passo (1), o professor adiciona o vídeo, seja um vídeo gravado ou de outro autor. No passo (2), o professor deve selecionar quais atividades serão utilizadas na aula. Em um ambiente sem o EVGAS, esse passo é feito manualmente. Entretanto, o plugin auxilia o professor ao requisitar automaticamente a classificação do vídeo para o Serviço e listar tags e atividades resultantes relacionados ao vídeo. Ainda neste passo, o professor define os elementos de gamificação que serão utilizados, assim como seus parâmetros. A Figura 4 ilustra, através de um mockup, a página de personalização da gamificação.

Os elementos de gamificação oferecidos pelo plugin são: pontos de experiência, badges, troféus e tabela de ranking. Por padrão, o plugin define cada atividade com o valor de 10 pontos, mas permite que o professor personalize esse valor. Esse elemento visa incentivar a resolução do maior número de tarefas para alcance dos melhores níveis de pontuação. O professor pode também definir badges e troféus que o aluno pode ganhar ao demonstrar certos 
comportamentos e alcançar objetivos específicos definidos pelo professor. Esses elementos buscam incentivar comportamentos e ações dos alunos, recompensando-os por suas conquistas. O ranking é criado a partir dos pontos de experiência adquiridos pelos alunos, ordenando-os de maneira não-crescente. O objetivo do ranking é incentivar os alunos a competirem entre si e realizarem mais atividades.

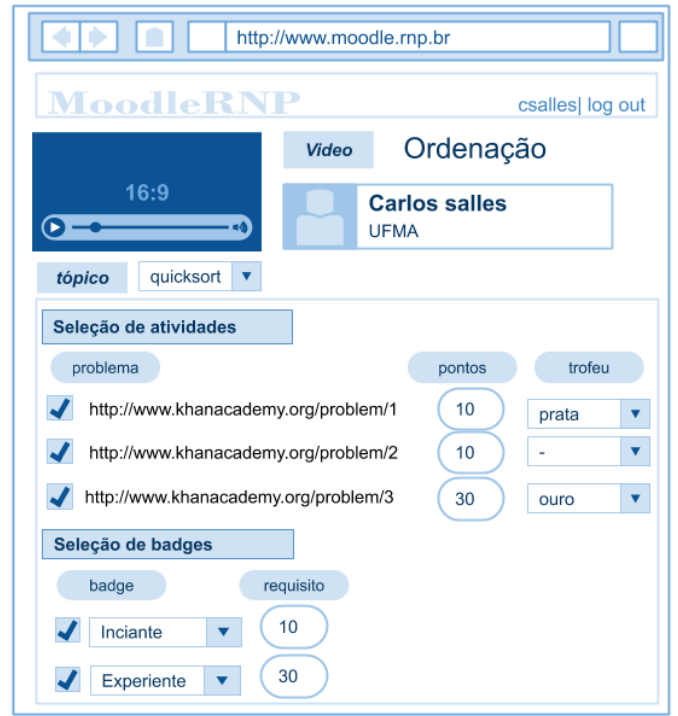

Figura 4: Adaptação da Gamificação pelo Professor

O passo (3) consiste no acompanhamento dos alunos na resolução das atividades. O plugin EVGAS fornece a visualização dos dados das atividades realizadas pelos alunos. Essa visualização inclui a confirmação individual dos alunos na resolução das atividades nos repositórios e os resultados dos elementos de gamificação incluídos. Para o professor, além da visualização dos resultados, o plugin permite a visualização por tópico selecionado. Assim, ele tem informações dos tópicos mais e menos explorados pelos alunos, podendo adaptar suas aulas para reforçar aqueles assuntos com poucas atividades realizadas.

\subsection{Serviço EVGAS}

O serviço EVGAS é o responsável receber requisições do plugin presente em instâncias de AVA. Ele possui três endpoints principais.

O primeiro consiste no (1) endpoint de classificação de vídeo. Ele recebe uma videoaula e extrai os possíveis tópicos abordados. $\mathrm{O}$ resultado da classificação é uma lista de tags que é apresentada ao professor pelo plugin para seleção. A partir de então, o plugin pode utilizar o (2) endpoint de busca de atividades por tags. Esse endpoint realiza uma busca por atividades relacionadas às tags em plataformas como o Khan Academy, UVA Onlinefudge entre outros, que são utilizadas aqui como repositórios de atividades. $\mathrm{O}$ resultado dessa busca é uma lista de atividades apresentada ao professor para possível utilização na sua aula.

O último consiste no (3) endpoint de classificação de vídeo. Ele realiza a busca pela resolução das atividades nos repositórios usados dado um aluno. Ele utiliza ids dos alunos nos repositórios de atividades. Logo o serviço verifica na plataforma da atividade se estes de fato fizeram as atividades selecionadas pelo professor. Assim, o serviço mantém um registro de todos os alunos e das atividades realizadas por estes. Este registro é utilizado para o acompanhamento do progresso dos alunos pelo professor.

\section{CONSIDERAÇÕES FINAIS}

Nossa Visão de Futuro para Deep Learning Aplicado a Educação consiste no uso dessas técnicas para melhorar o engajamento de alunos em AVAs. Nesse sentido consideramos duas frentes de trabalho: (1) classificação de vídeos; e (2) recomendação de conteúdo. Acreditamos que o primeiro permite uma melhor navegação e busca de conteúdos educacionais. Por outro lado, o segundo permite recomendar conteúdos que mantenham o usuário ativo e engajado na plataforma de ensino.

Este artigo discute a proposta de um DLaS (Deep Learning as a Service) chamado EVGAS (Educational Video Gamification As a Service). Ele aborda a frente de recomendação de conteúdo citada acima. E consiste em um serviço para gamificação de vídeos educacionais presentes em AVAs. Primeiro, ele utiliza técnicas de Deep Learning para realizar a classificação dos vídeos. Em seguida, ele acessa repositórios de atividades como o Khan Academy e UVA Onlinefudge para realizar a recomendação. Ele cria uma lista de atividades recomendadas para inserção no AVA e permite a gamificação do curso de forma pré-definida ou adaptada pelo professor.

Ressaltamos que o EVGAS é um projeto de pesquisa em andamento. Logo, este trabalho apresenta como resultados, a discussão de requisitos de alto nível e um mockup da página de gamificação pelos EVGAS. Com trabalhos futuros, visualizamos a necessidade de definir a ontologia de tags de ensino e desenvolver um design participativo sobre a User Experience (UX) da ferramenta com professores.

\section{REFERÊNCIAS}

[1] Sebastian Deterding, Dan Dixon, Rilla Khaled, and Lennart Nacke. 2011. From game design elements to gamefulness: defining gamification. In Proceedings of the 15th international academic MindTrek conference: Envisioning future media environments. ACM, 9-15.

[2] Shawn Hershey, Sourish Chaudhuri, Daniel P. W. Ellis, Jort F. Gemmeke, Aren Jansen, Channing Moore, Manoj Plakal, Devin Platt, Rif A. Saurous, Bryan Seybold, Malcolm Slaney, Ron Weiss, and Kevin Wilson. 2017. CNN Architectures for Large-Scale Audio Classification. In International Conference on Acoustics, Speech and Signal Processing (ICASSP). https://arxiv.org/abs/1609.09430

[3] S Hitz and M Turnoff. 2005. Education goes digital; the evolution of online learning and the revolution in higher education. Association for Computing Machinery. Communication of the ACM 48 (2005), 10-59.

[4] Jie Hu, Li Shen, and Gang Sun. 2017. Squeeze-and-excitation networks. arXiv preprint arXiv:1709.01507 7 (2017).

[5] Karl M Kapp. 2012. The gamification of learning and instruction: game-based methods and strategies for training and education. John Wiley \& Sons.

[6] Ana Carolina Tomé Klock, Mayco Farias de Carvalho, Brayan Eduardo Rosa, and Isabela Gasparini. 2014. Análise das técnicas de Gamificação em Ambientes Virtuais de Aprendizagem. RENOTE 12, 2 (2014).

[7] Joey J Lee and Jessica Hammer. 2011. Gamification in education: What, how, why bother? Academic exchange quarterly 15, 2 (2011), 146.

[8] Javier Melero, Davinia Hernández Leo, and Josep Blat. 2012. A Review of Constructivist Learning Methods with Supporting Tooling in ICT Higher Education: Defining Different Types of Scaffolding. F. UCS 18, 16 (2012), 2334-2360.

[9] Antoine Miech, Ivan Laptev, and Josef Sivic. 2017. Learnable pooling with Context Gating for video classification. arXiv preprint arXiv:1706.06905 (2017).

[10] Kaoru Ota, Minh Son Dao, Vasileios Mezaris, and Francesco GB De Natale. 2017. Deep learning for mobile multimedia: A survey. ACM Transactions on Multimedia Computing, Communications, and Applications (TOMM) 13, 3s (2017), 34.

[11] Maria da Graça Brasil Rocha, Maria do Carmo Nicoletti, and Oscar Hipólito. 2015. Alguns Aspectos da Formação Graduada em Computação no Brasil. InterSciencePlace 1, 26 (2015)

[12] Gabe Zichermann and Christopher Cunningham. 2011. Gamification by design: Implementing game mechanics in web and mobile apps. "O’Reilly Media, Inc.". 